CHAPTER 17

\title{
CONNECTIVITY OF JULIA SETS FOR SINGULARLY PERTURBED RATIONAL MAPS
}

\author{
ROBERT L. DEVANEY \\ Dept. of Mathematics, Boston University, \\ Boston, MA 02215, USA \\ ELIZABETH D. RUSSELL \\ Dept. of Mathematics, Western New England University, \\ Springfield, MA 01119, USA
}

\begin{abstract}
In this paper we consider the family of rational maps of the form $F_{\lambda}(z)=z^{n}+\lambda / z^{n}$ where $n \geq 2$. It is known that there are two cases where the Julia sets of these maps are not connected. If the critical values of $F_{\lambda}$ lie in the basin of $\infty$, then the Julia set is a Cantor set. And if the critical values lie in the preimage of the basin surrounding the pole at 0 , then the Julia set is a Cantor set of concentric simple closed curves around the origin. We prove in this paper that, in all other cases, the Julia set of $F_{\lambda}$ is a connected set.
\end{abstract}

1. Introduction . . . . . . . . . . . . . . . . . . . . . . . . . . . . .

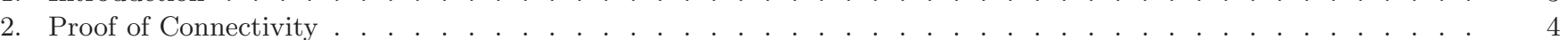

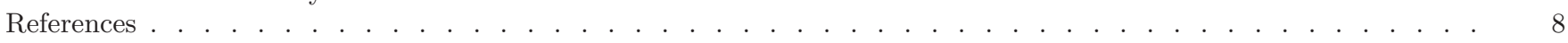

\section{Introduction}

Our goal in this paper is to describe the connectivity properties of Julia sets of functions in the family of rational maps of the Riemann sphere given by

$$
F_{\lambda}(z)=z^{n}+\frac{\lambda}{z^{n}}
$$

where $n \geq 2$ and $\lambda \in \mathbb{C}$. These maps are called singular perturbations since, when $\lambda=0$, the map is just $z \mapsto z^{n}$ and so the dynamics are completely understood. But when $\lambda \neq 0$, the degree of the map jumps from $n$ to $2 n$ and the dynamical behavior becomes much more complicated.

Despite the high degree of these maps, there are a number of similarities with complex polynomials of degree 2. For example, near $\infty$, the map is conjugate to $z \mapsto z^{n}$, so there is an immediate basin of attraction of $\infty$ which we denote by $B_{\lambda}$. Unlike polynomials, however, the full basin of $\infty$ is never a simply connected set. There is a pole at the origin and there are many parameters for which there is an open set surrounding 0 that is mapped $n$-to-one onto $B_{\lambda}$. If this set is disjoint from $B_{\lambda}$, we call this set the trap door and denote it by $T_{\lambda}$. If the orbit of any point eventually enters $B_{\lambda}$, it must do so by passing through the trap door.

In another analogy with quadratic polynomials, there is essentially only one free critical orbit for $F_{\lambda}$. One checks easily that there are $2 n$ free critical points given by $c_{\lambda}=\lambda^{1 / 2 n}$. (We call these points "free" critical points since $\infty$ is also a critical point, but it is fixed, and 0 is another also a critical 
point, but 0 is mapped directly to $\infty$.) Despite the large number of critical points, there are only two critical values $v_{\lambda}= \pm 2 \sqrt{\lambda}$. Exactly $n$ of the critical points are mapped to one critical value and the other $n$ are mapped to the other critical value. By symmetry, however, there is essentially only one free critical orbit, since, if $n$ is odd, the orbits of $\pm v_{\lambda}$ behave symmetrically under $z \mapsto-z$, whereas if $n$ is even, both $\pm v_{\lambda}$ are mapped to the same point by $F_{\lambda}$.

For quadratic polynomials, there is a wellknown dichotomy: if the critical orbit escapes to $\infty$, the Julia set is a Cantor set, while if the critical orbit does not escape, the Julia set is a connected set. In analogy with this, there is a somewhat different situation for $F_{\lambda}$; in this case we have an "escape trichotomy." In [6], the following theorem was proved:

Theorem. For the family $F_{\lambda}$ :

(1) If one of the critical values lies in $B_{\lambda}$, then $J\left(F_{\lambda}\right)$ is a Cantor set and $F_{\lambda} \mid J\left(F_{\lambda}\right)$ is conjugate to the one-sided shift on $2 n$ symbols.

(2) If one of the critical values lies in $T_{\lambda}$ (and, by hypothesis, $T_{\lambda}$ is disjoint from $\left.B_{\lambda}\right)$, then $J\left(F_{\lambda}\right)$ is a Cantor set of concentric simple closed curves (quasicircles).

(3) If one of the critical values lies in some preimage of $T_{\lambda}$, then $J\left(F_{\lambda}\right)$ is a Sierpiński curve.

We remark that part two of this theorem was first proved by McMullen [7]. It turns out that this kind of Julia set does not occur when $n=2$; indeed, the case $n=2$ is very different from (and much more complicated than) the case $n>2$. See [3].

A Sierpiński curve is any planar set that is homeomorphic to the well-known Sierpiński carpet fractal. The first such set to arise as a Julia set was found by Milnor and Tan Lei [9]. Since then, it has been shown that Sierpiński curves arise in many different ways as Julia sets [1,2]. See Fig. 1 for examples of the three different cases arising in the family $z^{4}+\lambda / z^{4}$.

In this paper, our goal is to strengthen the Escape Trichotomy Theorem by proving the following:

Theorem. If the critical values of $F_{\lambda}$ do not lie in either $B_{\lambda}$ or $T_{\lambda}$, then the Julia set of $F_{\lambda}$ is always a connected set.

So, just as in the case of quadratic polynomials, for these rational maps, either the Julia set is connected or else it is disconnected and consists of uncountably many disjoint components. In this latter case, as mentioned above, either the Julia set is a
Cantor set or else it is a Cantor set of simple closed curves. In Fig. 2, we display the parameter plane for the case $n=4$. The external region is the Cantor set locus. The central disk is the region where the Julia set is a Cantor set of simple closed curves; this region is called the McMullen domain. The complement of the Cantor set locus and the McMullen domain is the connectedness locus since we shall show in this paper that all of these parameters are associated to maps whose Julia sets are connected sets. Those parameters drawn from the red disks in the connectedness locus are Sierpiński curve Julia sets alluded to in the escape trichotomy.

Dedication. This paper is dedicated to Leon Chua, who has made the International Journal of Bifurcation and Chaos into one of the most influential journals in the field of dynamical systems. The first author of this paper has enjoyed working with him on the editorial board of this journal for many years.

\section{Proof of Connectivity}

Our goal in this section is to prove that the Julia sets drawn from the connectedness locus are always connected sets. In order to prove this, we need to show that all of the Fatou components of $F_{\lambda}$ are simply connected sets. There are thus only two ways that this could not happen; either there is a Herman ring or else there is an infinitely connected attracting or parabolic basin in the Fatou set. See [8]. If the Fatou set only has preimages of $B_{\lambda}$ or simply connected attracting or parabolic basins or Siegel disks, then the Julia set is the complement of infinitely many open and disjoint disks and hence is a connected set.

We first show that there is no Herman ring in the Fatou set. As a remark, this was shown in [11], but the proof we give here is much simpler. This is the easiest case of the Theorem.

First suppose we have a periodic Herman ring. Recall that a Herman ring is an open annular region on which some iterate of $F_{\lambda}$ is conjugate to an irrational rotation. At least one of the iterates of this ring must surround the origin, for if not, $F_{\lambda}$ would map the interior component of each Herman ring to the corresponding interior component of its image. But then the family of iterates of $F_{\lambda}$ would be normal on each such ring together with its bounded interior component. Hence there would be no interior boundary of the Herman ring in the Julia set 


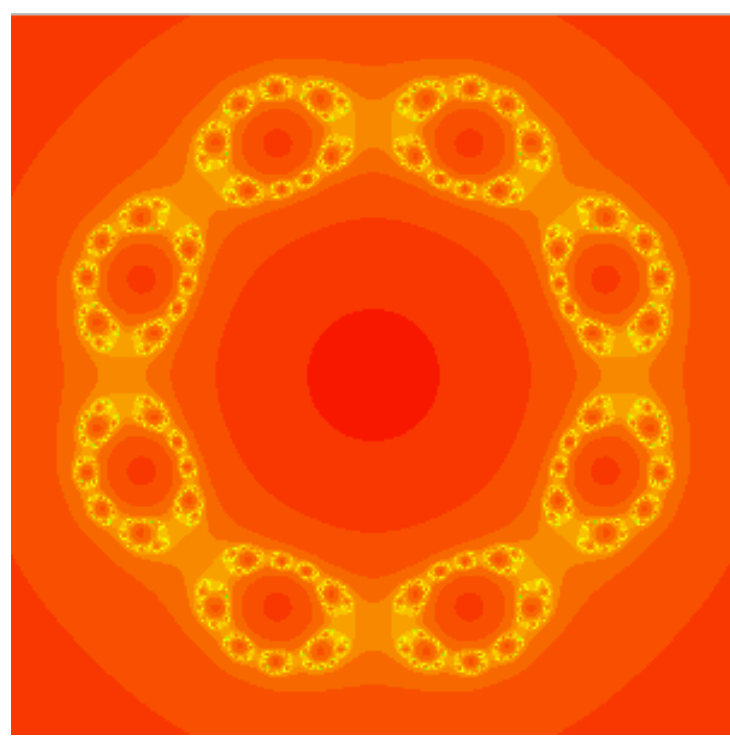

$\lambda=0.23$

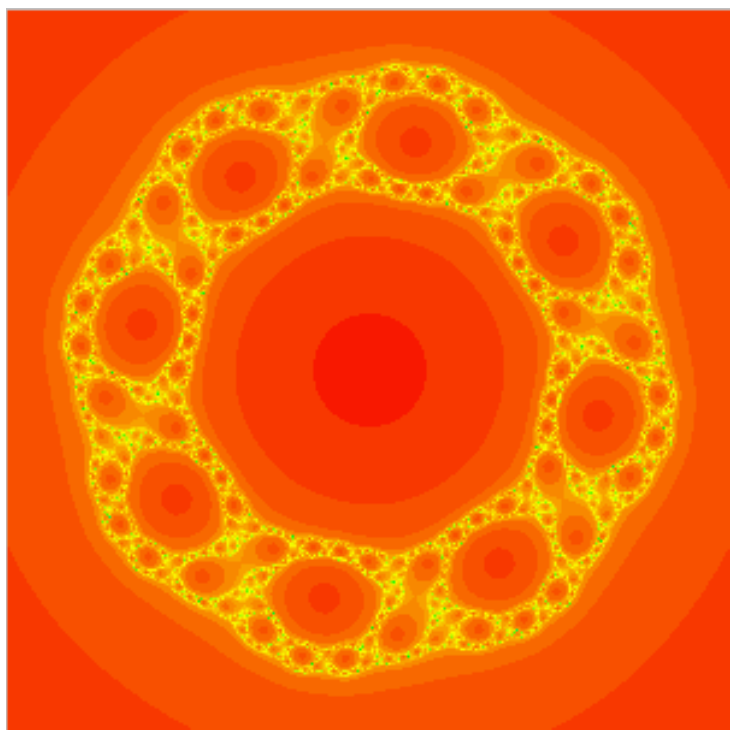

$\lambda=0.04$

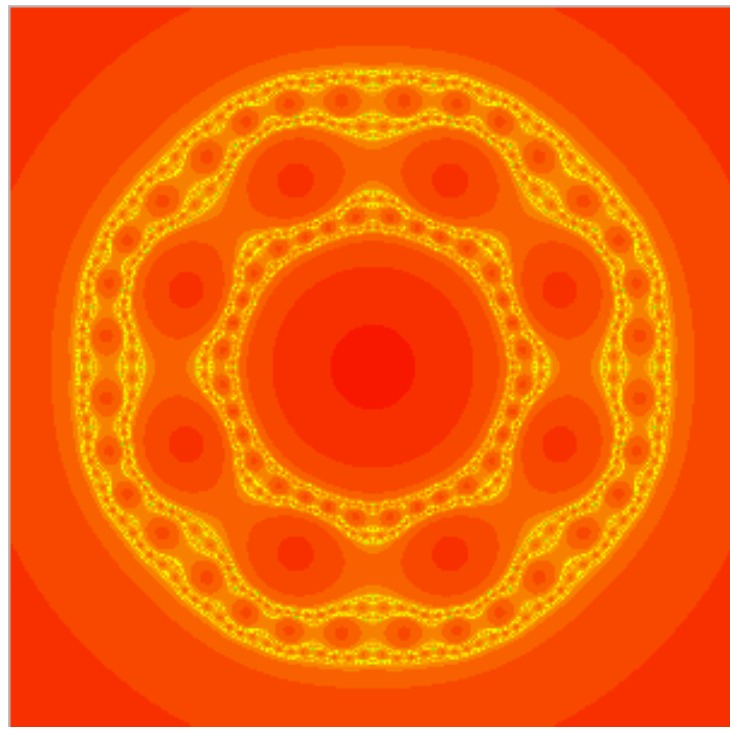

$\lambda=-0.125 i$

Fig. 1. Some Julia sets for $z^{4}+\lambda / z^{4}$ : if $\lambda=0.23, J\left(F_{\lambda}\right)$ is a Cantor set; if $\lambda=0.04, J\left(F_{\lambda}\right)$ is a Cantor set of circles; and if $\lambda=-0.125 i, J\left(F_{\lambda}\right)$ is a Sierpiński curve.

and the Fatou component would be the entire disk, not an annulus.

So let $R$ be a Herman ring that surrounds the origin. We have that the Julia set of $F_{\lambda}$ is symmetric under rotation by $2 \pi / 2 n$, i.e., it has $2 n$-fold symmetry. This follows from the fact that $F_{\lambda}\left(e^{\pi i / n}\right)$ $=-F_{\lambda}(z)$ and, as we observed above, the orbits of $\pm z$ are either symmetric under $z \mapsto-z$ (when $n$ is odd) or $F_{\lambda}(z)=F_{\lambda}(-z)$ when $n$ is even. In particular, all of the points of the form $e^{\pi i / n} z$ are mapped to the same point.

So assume that we have a Herman ring $R$ surrounding the origin. Then $R$ must be symmetric under the rotation $z \mapsto e^{\pi i / n} z$. But then, by the above, the map cannot be one-to-one on $R$, so we have no such Herman ring. This eliminates the first possibility of a non-simply connected component in the Fatou set.

Now suppose that the map has a multiply connected attracting or parabolic basin. Then it is well known [8] that this domain must in fact be infinitely connected. As above, at least one iterate of this domain must surround the origin. To show that this cannot happen when $\lambda$ is in the connectedness locus, we shall construct Cantor necklaces in the dynamical plane. The boundaries of these necklaces 


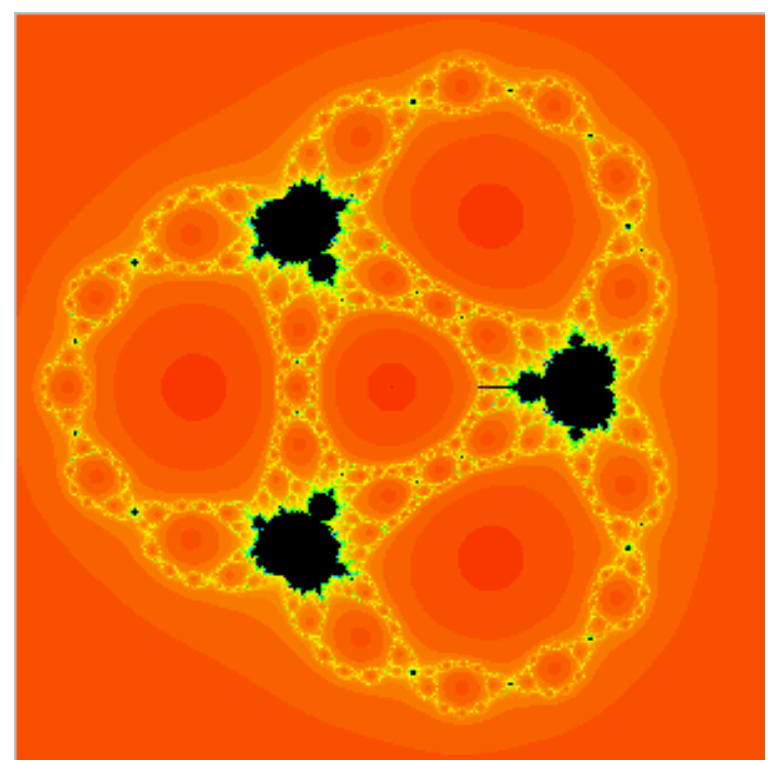

Fig. 2. The parameter plane when $n=4$.

will be closed sets that connect the boundary of $B_{\lambda}$ to the boundary of $T_{\lambda}$ and lie in the Julia set. This will show that there is no such infinitely connected Fatou component surrounding the origin.

For simplicity, we shall concentrate on the case $n=2$; the cases where $n>2$ are handled in a similar manner; we will discuss the minor modifications for these cases at the end of this section. To define the Cantor necklace, we let $\Gamma$ denote the Cantor middlethirds set in the unit interval $[0,1]$. We regard this interval as a subset of the real axis in the plane. For each open interval of length $1 / 3^{n}$ removed from the unit interval in the construction of $\Gamma$, we replace this interval by an open disk of diameter $1 / 3^{n}$ centered at the midpoint of the removed interval. Thus the boundary of this open disk meets the Cantor set at the two endpoints of the removed interval. We call the resulting set the Cantor middle-thirds necklace. See Fig. 3. Any set homeomorphic to the Cantor middle-thirds necklace is called a Cantor necklace. We do not include the boundary of the open disks in the Cantor necklace for the following technical reason: it is sometimes difficult in practice to verify that these bounding curves are simple closed curves.

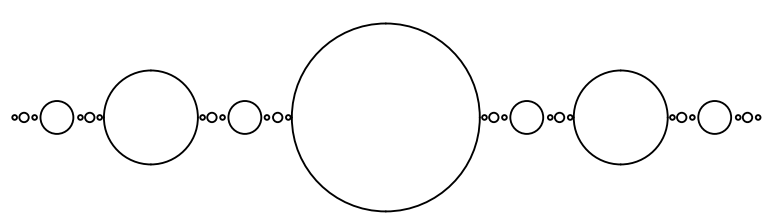

Fig. 3. The Cantor middle-thirds necklace.
Now let $\lambda=|\lambda| e^{i \eta}$. For the rest of this paper, we will consider only the case where $0<\eta<2 \pi$. When $\lambda$ is positive and real, it is known that $F_{\lambda}$ is conjugate in the right half plane to a polynomial of the form $z^{2}+c$ where $c \in \mathbb{R}^{+}$. See [4]. The boundary of this quadratic like Julia set then lies in the Julia set of $F_{\lambda}$ and stretches from $B_{\lambda}$ to $T_{\lambda}$ and so no Fatou domain can surround the origin in this case. We remark that all of the results below concerning Cantor necklaces also hold when $\eta=0$, but the arguments are slightly different.

When $|\lambda|<1$, we have the following estimate of the size of $B_{\lambda}$.

Lemma. (The Escape Criterion). Suppose $|\lambda|<1$ and $|z| \geq 2$. Then $z \in B_{\lambda}$, so the Julia set is contained in the open disk $|z|<2$.

Proof. If $|z| \geq 2$, then we have

$$
\left|F_{\lambda}(z)\right| \geq|z|^{2}-\frac{|\lambda|}{|z|^{2}} \geq 2|z|-\frac{1}{4}>\frac{3}{2}|z|
$$

Inductively, we find

$$
\left|F_{\lambda}^{n}(z)\right| \geq\left(\frac{3}{2}\right)^{n}|z|
$$

Therefore the orbit of any such $z$ tends to $\infty$ so all points on or outside the circle of radius 2 lie in $B_{\lambda}$.

As mentioned above, it is known that the Julia set is a Cantor set if $|\lambda| \geq 1$, so we also exclude this case for the remainder of this paper. Therefore we assume throughout that $\lambda=|\lambda| e^{i \eta}$ with $0<\eta<2 \pi$ and $|\lambda|<1$.

Recall that the critical points of $F_{\lambda}$ are given by $\lambda^{1 / 4}$. Therefore one of the critical points of $F_{\lambda}$ lies on the straight line through the origin given by $t \exp (i \eta / 4)$ with $t>0$. The image of this line lies along the straight line with argument $\theta=\eta / 2$, and $F_{\lambda}$ maps the line $t \exp (i \eta / 4)$ with $t>0$ in two-toone fashion over the portion of this image straight line that extends from the critical value $2 \sqrt{\lambda}$ whose argument is $\eta / 2$ out to $\infty$. Note that the image of this line is disjoint from the line itself since we have assumed that $0<\eta<2 \pi$. There is a second critical point of $F_{\lambda}$ lying on the line with argument $\theta=\eta / 4-\pi / 2$, and this line is mapped in two-to-one fashion to the opposite line $\theta=-\eta / 2$ exactly as in the above case.

Let $H_{\lambda}$ be the involution $\lambda^{1 / 2} / z$. Then we have $F_{\lambda}\left(H_{\lambda}(z)\right)=F_{\lambda}(z)$ so the Julia set of $F_{\lambda}$ is 


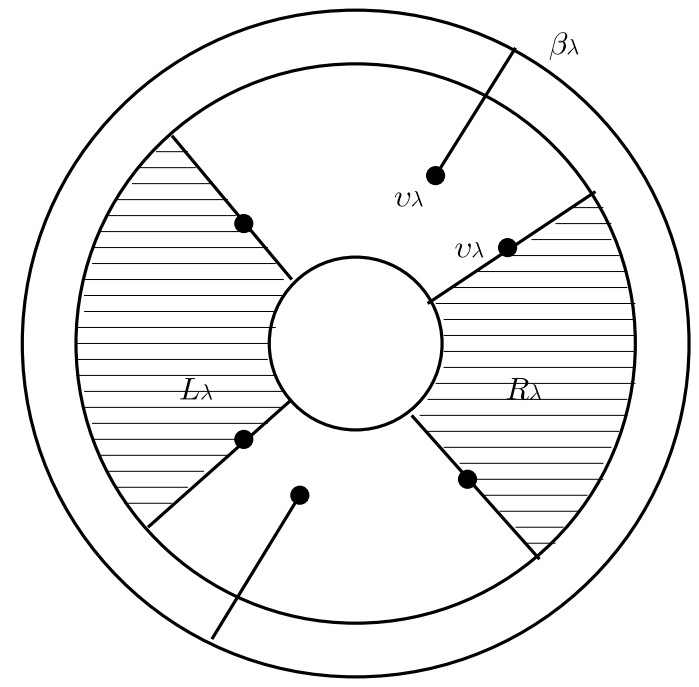

Fig. 4. $\quad R_{\lambda}$ and $L_{\lambda}$ and their image under $F_{\lambda}$, which is the interior of $\beta_{\lambda}$ minus the two segments connecting this circle to the critical values.

symmetric under $H_{\lambda}$. Note that $H_{\lambda}$ interchanges $B_{\lambda}$ and $T_{\lambda}$. By the escape criterion, we know that any point on or outside $r=2$ is mapped closer to $\infty$. Let $\beta_{\lambda}$ denote the image of this circle, so that $\beta_{\lambda} \subset B_{\lambda}$. Using the involution $H_{\lambda}$, there is a second circle, namely $r=|\lambda|^{1 / 2} / 2$, that is also mapped two-to-one onto $\beta_{\lambda}$.

Consider the open region $R_{\lambda}$ bounded by the rays $\theta=\eta / 4$ and $\theta=\eta / 4-\pi / 2$ and the two circular preimages of $\beta_{\lambda}$. The set $R_{\lambda}$ is a quarter of an annulus. Let $L_{\lambda}=-R_{\lambda}$. We call $R_{\lambda}$ (resp., $L_{\lambda}$ ) the right (resp., left) fundamental sector. These fundamental sectors are a pair of disjoint, open, simply connected regions in $\mathbb{C}$. Note that, for each $\lambda, R_{\lambda}$ lies in the right half plane $\operatorname{Re} z>0$, while $L_{\lambda}$ lies in the left half plane. See Fig. 4.

Proposition. $F_{\lambda}$ maps each of the fundamental sectors in one-to-one fashion onto the open set $\mathcal{O}$ bounded by $\beta_{\lambda}$ minus the portions of the two straight lines $\theta= \pm \eta / 2$ extending from the critical values $\pm v_{\lambda}$ to $\beta_{\lambda}$. So the image of each of these fundamental sectors contains the closures of both $R_{\lambda}$ and $L_{\lambda}$ in its interior.

Proof. The images of the straight rays bounding $R_{\lambda}$ and $L_{\lambda}$ are contained in the rays $\theta= \pm \eta / 2$, both of which lie outside these sectors. The image of the outer circular boundary of each fundamental sector is exactly one-half of $\beta_{\lambda}$, while the inner boundary of each sector is mapped to the opposite half of $\beta_{\lambda}$. Hence each fundamental sector is mapped onto the open disk bounded by $\beta_{\lambda}$ minus the two portions of the rays $\theta= \pm \eta / 2$ lying beyond the critical values. This set is $\mathcal{O}$. By fourfold symmetry, this map must be one-to-one on each fundamental sector.

Since $F_{\lambda}$ maps the union of the fundamental sectors strictly outside itself, most points in $R_{\lambda} \cup L_{\lambda}$ have orbits that leave this set at some iterate. Let $\Gamma_{\lambda}$ be the set of points whose orbits remain for all iterations in $R_{\lambda} \cup L_{\lambda}$. Then we have:

Proposition. The set $\Gamma_{\lambda}$ is a Cantor set and $F_{\lambda} \mid \Gamma_{\lambda}$ is conjugate to the one-sided shift on two symbols.

Proof. By the previous result, each of the fundamental sectors is mapped in one-to-one fashion onto the open region $\mathcal{O}$ that properly contains $R_{\lambda} \cup L_{\lambda}$ in $\mathbb{C}$. So we have a pair of well-defined inverses $G_{0}$ (resp., $G_{1}$ ) of $F_{\lambda}$ that map $\mathcal{O}$ into $R_{\lambda}$ (resp., $L_{\lambda}$ ). Standard arguments then show that these inverses are contractions in the Poincaré metric on $\mathcal{O}$. Then, for any one-sided sequence $\left(s_{0} s_{1} s_{2} \ldots\right)$ of 0 's and 1's, the set

$$
\bigcap_{j=0}^{\infty} G_{s_{0}} \circ \ldots \circ G_{s_{j}}(\mathcal{O})
$$

is a unique point and the map that takes the sequence $\left(s_{0} s_{1} s_{2} \ldots\right)$ to this point defines a homeomorphism between the space of one-sided sequences of 0's and 1's endowed with the usual topology and $\Gamma_{\lambda}$. Hence $\Gamma_{\lambda}$ is a Cantor set and we have that $F_{\lambda} \mid \Gamma_{\lambda}$ is conjugate to the one-sided shift on two symbols.

Now suppose, in addition, that the critical values do not lie in $B_{\lambda}$. So $B_{\lambda}$ is a simply connected open set. Our goal now is to construct a Cantor necklace in the dynamical plane. The Cantor set portion of the necklace will be the set $\Gamma_{\lambda}$ constructed above, whereas the open disks will be certain of the preimages of the basin of $\infty$ that lie in $R_{\lambda}$ and $L_{\lambda}$.

To construct the necklace, we have that $F_{\lambda}$ is conjugate to $z^{2}$ in $B_{\lambda}$. Hence there are a pair of external rays in $B_{\lambda}$ that correspond to the rays of angles 0 and $\pi$ in the basin of $\infty$ for $z^{2}$. It is known [10] that the external ray of angle 0 limits on the fixed point $p_{\lambda}$ that lies in $R_{\lambda} \cap \partial B_{\lambda}$ and the external ray of angle $1 / 2$ limits on its preimage $-p_{\lambda}$ in $L_{\lambda}$. Let $\pm q_{\lambda}$ be the preimages of $-p_{\lambda}$ in $L_{\lambda}$ and $R_{\lambda}$. Then there is a unique curve in $T_{\lambda}$ passing through 0 and connecting $q_{\lambda}$ to $-q_{\lambda}$ that 
is mapped onto the external ray connecting $-p_{\lambda}$ to $\infty$. Since $T_{\lambda}$ is open and simply connected, we may define a homeomorphism that takes $T_{\lambda}$ onto a disk centered at $1 / 2$ on the real line and having radius $1 / 6$. This is the central disk in the Cantor middlethirds necklace. Moreover, we may arrange that this homeomorphism extends to the points $\pm q_{\lambda}$ in $\partial T_{\lambda}$ so that the point $-q_{\lambda}$ is sent to $1 / 3$ and the point $q_{\lambda}$ is sent to $2 / 3$.

Now consider the preimages of the trap door. Since the critical values do not lie in the trap door, there are four preimages of this set, but only two of them lie in the fundamental sectors by the fourfold symmetry. The other two lie in the complementary sectors. These give a pair of simply connected open sets that contain the preimages of $\pm q_{\lambda}$ in their boundaries; one of these sets lies in $R_{\lambda}$, the other lies in $L_{\lambda}$. These may be mapped homeomorphically to the open disks in the Cantor middle-thirds necklace whose diameter is $1 / 9$ with the property that an extension of this homeomorphism takes the preimages of $\pm q_{\lambda}$ to the corresponding endpoints of the Cantor middle thirds set. We then continue in this fashion by taking appropriate preimages of the trap door under compositions of $G_{0}$ and $G_{1}$ and defining a homeomorphism between these preimages and the appropriate disk in the Cantor middle-thirds necklace. Note that the symbolic dynamics described above dictates which preimages correspond to which disks. As above, we extend this homeomorphism to the two special boundary points that eventually map to $p_{\lambda}$. This defines the homeomorphism on a set whose image is then the union of the disks in the middle-thirds necklace together with the corresponding endpoints in their boundaries. By the symbolic dynamics, these preimages of $p_{\lambda}$ are dense in the Cantor set $\Gamma_{\lambda}$, so we may extend this homeomorphism to all of the remaining points in the Cantor set. This then gives a homeomorphism between the points in $\Gamma_{\lambda}$ together with the preimages of the trap door in $R_{\lambda} \cup L_{\lambda}$ and the Cantor middle thirds necklace. We have proved:

Proposition. Suppose that the critical values of $F_{\lambda}$ do not lie in $B_{\lambda}$ or $T_{\lambda}$ and that $0<\operatorname{Arg} \lambda<2 \pi$. Then the set $\Gamma_{\lambda}$ together with the preimages of $B_{\lambda}$ under compositions of the maps $G_{0}$ and $G_{1}$ forms a Cantor necklace.

Note that the boundary of the Cantor necklace consists of points that lie in either the Cantor set portion of the necklace or else on the boundaries of the preimages of the trap door. Thus the boundary of the necklace lies in the Julia set and therefore provides a closed set that connects the boundaries of $T_{\lambda}$ and $B_{\lambda}$. This proves that there are no multiply connected Fatou components of $F_{\lambda}$ and hence that the Julia set is connected when $\pm v_{\lambda}$ do not lie in $B_{\lambda}$ or $T_{\lambda}$.

For the case $n>2$, there is a similar escape criterion which is easy to compute and we also have the involution symmetry, now given by $H_{\lambda}(z)=\lambda^{1 / n} / z$. The construction of the Cantor necklace is similar; the only difference is that we have $2 n$ fundamental sectors in this case. Choosing the two that straddle the real axis then provides the region in which the necklace exists. We remark that, when $n>2$ a much more complicated object known as a Cantor web may be constructed. See [5].

\section{References}

[1] Blanchard, P., Devaney, R. L., Look, D. M., Moreno Rocha, M., Seal, P., Siegmund, S., Uminsky, D. Sierpiński Carpets and Gaskets as Julia Sets of Rational Maps. In Dynamics on the Riemann Sphere. European Math Society (2006), 97-119.

[2] Devaney, R. L. A Myriad of Sierpiński Curve Julia Sets. In Difference Equations, Special Functions, and Orthogonal Polynomials, World Scientific (2007), 131-148.

[3] Devaney, R. L. Dynamics of $z^{n}+C / z^{n}$; Why the Case $n=2$ is Crazy. To appear in Conformal Dynamics and Hyperbolic Geometry, American Math Society, Contemporary Math.

[4] Devaney, R. L. Baby Mandelbrot Sets Adorned with Halos in Families of Rational Maps. In Complex Dynamics: Twenty-Five Years After the Appearance of the Mandelbrot Set. American Math Society, Contemporary Math, 396, 2006, 51-62.

[5] Devaney, R. L. Cantor Webs in the Parameter and Dynamical Planes of Rational Maps. Fields Institute Communications 53 (2008), 105-123.

[6] Devaney, R. L., Look, D. M., and Uminsky, D. The Escape Trichotomy for Singularly Perturbed Rational Maps. Indiana University Mathematics Journal 54 (2005), 1621-1634.

[7] McMullen, C. The Classification of Conformal Dynamical Systems. Current Developments in Mathematics. International Press, Cambridge, MA, (1995) 323-360.

[8] Milnor, J. Dynamics in One Complex Variable. Third Edition. Annals of Mathematics Studies. Princeton University Press, (2006).

[9] Milnor, J. and Tan Lei. A "Sierpiński Carpet" as Julia Set. Appendix F in Geometry and Dynamics 
of Quadratic Rational Maps. Experiment. Math. 2 (1993), 37-83.

[10] Petersen, C. and Ryd, G. Convergence of Rational Rays in Parameter Spaces, The Mandelbrot set: Theme and Variations, London Mathematical
Society, Lecture Note Series 274, Cambridge University Press, 161-172, 2000.

[11] Xiao, Y. and Qiu, W. The Rational Maps $F_{\lambda}(z)=$ $z^{m}+\lambda / z^{d}$ Have No Herman Rings. Proc. Indian Acad. Sci. 120 2010, 403-407. 